\title{
Review of imaging solutions for integrated quantitative immunohistochemistry in the Pathology daily practice
}

\author{
Marcial García Rojoํㅜ, Gloria Bueno², Janina Slodkowska ${ }^{3}$ \\ 1Pathology Department, Hospital General de Ciudad Real, Ciudad Real, Spain \\ ${ }^{2}$ Engineering School, Universidad de Castilla-La Mancha, Ciudad Real, Spain \\ ${ }^{3}$ Military Institute of the Health Services, Warsaw, Poland
}

\begin{abstract}
Immunohistochemistry (IHC) plays an essential role in Pathology. In order to improve reproducibility and standardization of the results interpretation, IHC quantification methods have been developed. IHC interpretation based in whole slide imaging or virtual microscopy is of special interest. The objective of this work is to review the different computerbased programs for automatic immunohistochemistry and Fluorescence In Situ Hybridization (FISH) evaluation. Scanning solutions and image analysis software in immunohistochemistry were studied, focusing especially on systems based in virtual slides. Integrated scanning and image analysis systems are available (Bacus TMAScore, Dako ACIS III, Genetix Ariol, Aperio Image Analysis, 3DHistech Mirax HistoQuant, Bioimagene Pathiam). Other image analysis software systems (Definiens TissueMap, SlidePath Tissue Image Analysis) can be applied to several virtual slide formats. Fluorescence is the preferred approach in HistoRx AQUA, since it allows for a better compartmentalization of signals. Multispectral imaging using CRi Nuance allows multiple antibodies immunohistochemistry, and different stain unmixing. Most current popular automated image analysis solutions are aimed to brightfield immunohistochemistry, but fluorescence and FISH solutions may become more important in the near future. Automated quantitative tissue microarrays (TMA) analysis is essential to provide high-throughput analysis. Medical informatics standards in images (DICOM) and workflow (IHE) under development will foster the use of image analysis in Pathology Departments.
\end{abstract}

Key words: image analysis, digital slide, virtual microscopy, immunohistochemistry, fluorescence.

\section{Introduction}

Despite emerging technologies, from histochemical stains to molecular assays at the DNA, RNA, and protein levels, in evaluation of the basis of diseases, histopathology studies remain the gold standard.

In this context, immunohistochemistry (IHC) plays an essential role in the in situ assessment of protein expression, and also complements morphologic information with molecular information, and predicts a response to targeted therapy. New demands on the quality, reproducibility, and accuracy of this technology are claimed [1].

Taylor and Levenson stressed the importance that an immunohistochemical IHC stain is strictly analogous to an enzyme-linked immunosorbent assay (ELISA) test

Correspondence: M. Garcia Rojo, Coordinator of Information Systems, Servicio de Anatomia Patologica, Hospital General de Ciudad Real, Calle Tomelloso s/n. 13005 Ciudad Real, Spain; marcial@cim.es performed in the clinical laboratory; ELISA tests are widely recognized as being truly quantitative. Therefore, immunohistochemistry allows also for quantification [2]. These authors describe validation, proficiency testing and quality assurance in immunohistochemistry, considering the different phases of the process: pre-analytical (specimen handling, fixation, paraffin embedding, sectioning, and deparaffination), analytical (antigen retrieval, staining), and post-analytical (reading of results or scoring and reporting) [2].

IHC has become a standard assay in Pathology, but it often lacks reproducibility and standardization in the essential steps like fixation conditions, specimen pretreatment, reagents, detection methods, and interpretation of results. IHC interpretation may be standardized through the use of quantitative methods, using current digital imaging technology, avoiding the use of 3- or 4-point subjective scoring methods.

Another approach for standardization is the use of tissue microarrays (TMAs) allowing all tissues to be exposed to identical conditions. However, there are 
other steps difficult to control, such as prefixation treatment of a specimen in the operating room, inconsistent fixation modalities and times, as well as antigen oxidation [1].

During the last years a significant increase in the automation of procedures in Pathology has been noticed. This includes automated staining (conventional stains, histochemical techniques, immunostainers), and automated in situ hybridization systems; automatic slide preparation (coverslip, slide drying) and integrated slide and cassette labelling.

Standardization and automation are essential to foster translational research efforts in the study of new biomarkers for cancer care [3]. A group of the Yale University Medical School described the use fluorescence probes rather than the brown stain of conventional immunohistochemistry. This image analysis system (called AQUA) is an interesting solution for quantitative pathology to define a histologic region of interest, tissue and cellular compartmentalization, and signal localization [4].

Several image analysis applications for immunohistochemistry quantification, some of them commercially available and have received clearance from the US Food and Drug Administration (FDA), have proved that automatic quantification provides more reliable and uniform results than manual evaluation. Automated methods have the potential to be used as a computer aid for the immunohistochemical evaluation (e.g. human epidermal growth factor receptor 2 -HER2- expression) with the objective of increasing observer reproducibility [5].

The objective of this work is to review the different computer-based programs for automatic immunohistochemistry quantification.

\section{Materials and methods}

The analyzed variables were related to the image analysis in immunohistochemistry, focusing especially on systems based in virtual slides or while slide imaging. Available algorithms, tested immunomarkers, reliability, user interface, and integration with other information systems were studied.

From September to October 2008, a bibliographic search has been performed using the Pubmed service of the U.S. National Library of Medicine and the National Institutes of Health (http://www.ncbi.nlm.nih.gov/pubmed/). The main information was obtained by in situ demonstrations of product functionalities. Additional information was obtained by contacting the software vendors and visiting vendor websites (table 1).

\section{Results}

\section{Bacus TMAScore}

BLISS Slide Scanner is one of the first virtual slide systems that were available for automatic quantification in immunohistochemistry. The system scans the slide at low magnification $(\times 1.25)$ and then the user identifies a region of interest, which is then scanned at high magnification (up to $\times 63$ ). Immunohistochemical score is performed with Bacus IHC score software. The system allows obtaining measurements of 4 different stains, 21 Markovian texture, and 8 other morphologic parameters. Measurements are overlaid directly on the region of interest, which makes it easier for the user to interpret stains. Bacus laboratories, Inc is owned by Olympus America, Inc.

\section{ACIS III}

The Automated Cellular Imaging System (ACIS) III performs a scan of a $15 \times 15 \mathrm{~mm}$ area, $10 \times$, in 9 minutes. The available objectives are: $4 \times, 10 \times, 20 \times, 40 \times$, $60 \times$. It includes an automatic tissue finding algorithm. The system is based in the detection of levels of hue, saturation, and luminosity. The available IHC algorithms are nuclear, membrane, cytoplasmic, rare event, and TMA. ACIS III has FDA Clearance on HercepTest $^{\mathrm{TM}}$, ER and PR applications. A reporting tool integrates the pictures, graphics and multiple assay results. It allows for collaborative work using Dako EyeShare software in a network. An easy daily calibration makes the system reliable. ACIS III is distributed by Dako Denmark A/S and its affiliates. In March 2008, Carl Zeiss MicroImaging, Inc. acquired ACIS ${ }^{\circledR}$ Instrument Systems from Clarient, Inc.

\section{Ariol}

Genetix Applied Imaging Ariol is one of the pioneer image analysis systems available for both brightfield and fluorescent imaging. It allows quantification in IHC, FISH, and immunofluorescence. It has also been used in the study of micrometastasis, angiogenesis, DNA ploidy, and Tissue Micro Array. Ariol Microsight is a cellular rare event application. Hersight is the algorithm for HER-2/neu analysis. Other available algorithms are oestrogens receptor (ER), progesterone receptor (PR), and for the PathVysion ${ }^{\circledR}$ HER-2 DNA direct labelled FISH probes. CytoVision $\mathbb{}{ }^{\circ}$ is a solution for cytogenetics laboratory, including Karyotyper, a rapid metaphase finder and computer aided chromosome analysis system; CEP XY, to assess the effectiveness of bone marrow transplantation in oppositesex transplants; Fluorescence In-Situ Hybridization (FISH), Comparative Genomic Hybridization (CGH), SPOT AX, RxFISH Color Chromosome Analysis, and M-FISH.

\section{Aperio Image Analysis Toolbox}

All Aperio Scanscope scanning systems are shipped with a positive pixel count algorithm that may be used by pathologists for a measurement of area and staining 
Table 1. Image analysis systems available for virtual slides

\begin{tabular}{|c|c|}
\hline Product name & Manufacturer \\
\hline \multicolumn{2}{|c|}{ Virtual slide scanning and automatic image analysis } \\
\hline TM $\wedge$ score & $\begin{array}{l}\text { Bacus I.abs/Olympus America } \\
\text { http://www.olympusamerica.com/seg_section } / \text { seg_vm.asp }\end{array}$ \\
\hline ScanScope Image Analysis loolbox & $\begin{array}{l}\wedge \text { perio } \\
\text { htp://www.apcrio.com/imagcanalysis/image-analysis.asp }\end{array}$ \\
\hline MIRAX IlistoQuant & $\begin{array}{l}\text { 3DIlistech } \\
\text { http://www.3dhistech.com/en/article/quantitative-histology }\end{array}$ \\
\hline PАTHI $\triangle M^{T M}$ RUO & $\begin{array}{l}\text { Biolmagenc } \\
\text { http://www.bioimagene.com/pathiam.html }\end{array}$ \\
\hline ACIS & $\begin{array}{l}\text { Dako } \\
\text { htlp://www.dakousa.com/index/prod_scarch/prod_groups.htm?productareaid=43 }\end{array}$ \\
\hline Ariol & $\begin{array}{l}\text { Genetix } \\
\text { http://www.genetix.com/en/systems/ariol }\end{array}$ \\
\hline CytoVision & $\begin{array}{l}\text { Genelix } \\
\text { http://www.genetix.com/en/systems/cytovision }\end{array}$ \\
\hline \multicolumn{2}{|c|}{ Image analysis software and static picture solutions } \\
\hline TissueMap & $\begin{array}{l}\text { Delinicns } \\
\text { http://www.definiens.com/definiens-tissuemap_134_7_10.html }\end{array}$ \\
\hline Tissue Image $\wedge$ nalysis & $\begin{array}{l}\text { SlidePath } \\
\text { http://www.slidepath.com/php/products-imagcanalysis.php }\end{array}$ \\
\hline iCyte, I aser Scanning Cytometer & CompuCyte \\
\hline AQUA & $\begin{array}{l}\text { HistoRx } \\
\text { htlp://www.historx.com/AquaNew }\end{array}$ \\
\hline Nuance ${ }^{\mathrm{IM}}$ & $\begin{array}{l}\text { Cambridge Rescarch \& Instrumentation, Inc (CRi) } \\
\text { http://www.cri-inc.com/products/nuance.asp }\end{array}$ \\
\hline
\end{tabular}

intensity for two-colours, as well as to measure percentage positivity by area and average intensity of positive staining. Examples or applications are detection of percentage of vacuolization areas or necrotic tumour areas in a slide. Other algorithm are available from Aperio, some of them FDA cleared for in-vitro diagnosis, like HER2, and nuclear algorithms ER and $\mathrm{PR}$ in breast cancer.

TMALab is a software application to work with TMA, making easier spots locating and viewing, and automatic TMA analysis. Spot images can be exported to individual tiff files, for use in other applications. Other solutions available are Area Quantification, Cell Quantification, Microvessel Analysis, and Rare Event Detection. Genie ${ }^{\mathrm{TM}}$ is a histology pattern recognition tool that is useful for tumour areas detection, and then quantification algorithm application only to the tumour areas of interest. A colocalization tool is intended for colour deconvolution and colocalization analysis that allows counting of different cell subpopulations in the slides stained with two or more markers.

\section{Mirax HistoQuant}

Mirax scanners family generates high quality virtual slides that can be analyzed using 3DHistech MIRAX HistoQuant tools integrated in Mirax Viewer. This is one of the few available complete scanning and image analysis solutions for both brightfield and fluorescent slides. Image segmentation, nuclear analysis (counting nuclei and measuring the nuclear morphologic and densitometric features), cell membrane analysis and detection and measurement of glandular morphometric parameters are some examples of the available tools. FISH automatic analysis results can be expressed in a histogram. IH-Lab is a system to help scoring differently stained slides of a single specimen. TMA software module allows linking multiple TMA blocks of multiple slides together in one project.

\section{PATHIAM and TissueMine}

Bioimagene PATHIAM ${ }^{\mathrm{TM}}$ RUO (Pathology Image Analysis and Management Software) is for Research Use Only. It includes ER, PR, Ki-67, and p53 algo- 
rithms, and also morphological and intensity based algorithms. PATHIAM ${ }^{\text {TM }}$ IVD Imaging Software for HER2/neu is a semi-quantitative assessment tool for HER2 staining assessment which has been approved by FDA for in vitro diagnostic use in breast cancer.

TissueMine is a complete solution for acquisition, analysis, management, reporting and sharing of tissue images. Different TissueMine Packages are available. Bioimagene has developed 30 imaging algorithms available for IHC (antibody specific), FISH, Chromogenic In Situ Hybridization (CISH), morphometry (kidney), H+E (prostate cancer), cytology (Pap smear). Tissue Micro Arrays applications are also available.

\section{Definines TissueMap}

Definiens tissue analysis software includes TissueMap 3.0, an automatic nuclear, membrane, and cytoplasm biomarker quantisation software tool than can be used in whole tissue sections and tissue microarrays. Image analysis routines can be customized and user-defined. Digital pathology software detects and measures a wide variety of morphological parameters including spectral statistics, shape, position, texture and relationship to neighbouring structures.

\section{SlidePath}

SlidePath is specialized in digital pathology imaging software solutions. SlidePath's Tissue Image Analysis is aimed to obtain a high throughput image analysis for IHE stained slides, including three standard algorithms: membrane, nuclear and positive pixel. SlidePath Distiller is a web based information management system for sharing files and data between distributed collaborators. OpTMA module automates the TMA analysis process.

\section{HistoRx}

HistoRx AQUA services of quantitative biomarker analysis are available for licensing to researchers. The PM-2000 ${ }^{\text {TM }}$ image system obtains $2048 \times 2048$ pixels pictures. It is based on an Olympus BX51 fluorescent microscope that scans the whole slide at $4 \times$, and then an analysis can be performed at $10 \times, 20 \times, 40 \times$, or $60 \times$. Up to 5 fluorescent probes can be applied in the same tissue. AQUA compartmentalization of expression uses an exponential subtraction algorithm and pixelbased locale assignment. This approach has the following advantages: reducing the "out-of-focus" noise, improving the resolution, compartmentalization of expression, and measuring average intensity (AQUA score: pixel intensity/pixel area).

\section{Cambridge Research \& Instrumentation}

Cambridge Research \& Instrumentation, Inc (CRi) Nuance ${ }^{\mathrm{TM}}$ is a multispectral imaging system to quantify molecular markers even when they are co-localized in a single tissue section that can be applied to multiantibody immunohistochemistry co-localization. The system is able to collect different spectral images (from 450 to $950 \mathrm{~nm}$, covering most chromogens/ fluorophores) and is useful in chromogenic and fluorescence microscopy. In spectral imaging, a series of 3-20 images are taken from blue to the red (e.g. 420-700 $\mathrm{nm})$. The resulting image 'stack' is assembled in memory in such a way that a spectrum is associated with every pixel. This ability to sample the spectrum with many discrete wavelength regions spanning the visible wavelength range allows an accurate unmixing of multiple spatially colocalized chromogens.

\section{Discussion}

There are other available solutions for image analysis in immunohistochemistry, although they have not been included in this review because they are not based in whole slide imaging of pathology slides. Even so, it is worth mentioning that Ventana Image Analysis System (VIAS) has a chromogen separation tool to enhance visualization of cellular versus background staining and an auto-segmentation tool.

For a further detailed description of image analysis and scanning systems we refer the reader to previous works [6,7].

AQUA system was described by Bast et al (2005) as a Pathologist-free image analysis using fluorescence with the advantage of tissue compartmentalization that makes it easier to separate tumour from stroma. Cellular compartments can be detected using specific fluorescent probes, distinguish nuclei, cytoplasm, and membranes [3]. It has shown to be useful in prostate cancer diagnosis and breast cancer prognostic assessment of HER-2 and $\beta$-catenin. By using this technique it has been demonstrated that in situ quantitative measurement of HER2 stratifies breast tumours into three levels: normal, intermediate, and high HER2 expression. Unlike manual reading, an automated analysis revealed that patients with breast tumours expressing normal levels of HER2 also showed a significantly worse outcome. Therefore, both normal and high HER2 levels were associated with a worse outcome [8].

Most current popular automated image analysis solutions are aimed to brightfield immunohistochemistry. Several factors that affect performance of the imaging system should be considered to evaluate the reproducibility of obtained results. These can be classified into image acquisition factors: camera, illumination source, optical performance of the stains; and 


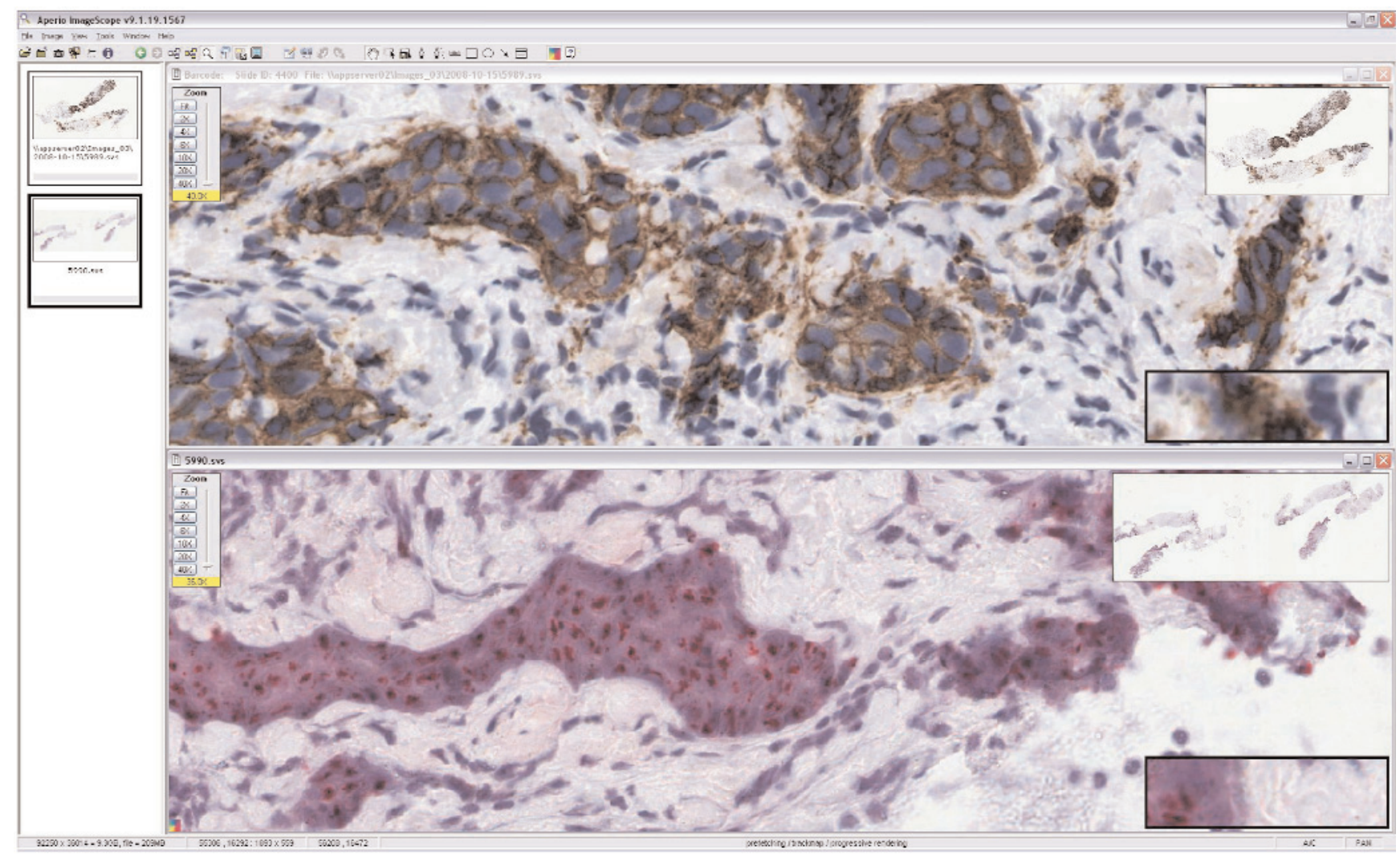

Fig. 1. Combined evaluation of HER-2 using both immunohistochemistry (top) and CISH (bottom) virtual slides.

post-image acquisition factors: a selection of the appropriate mathematical techniques applied to extract quantitative intensity and area measurements from the imaging data.

In our own experience, automated image analysis is more sensitive, but if not well parameterized, it tends to record a higher percentage of positivity (at least in $\mathrm{ER} / \mathrm{PR}$ ) than subjective rating. Colorimetric (RGB) or intensity (grey scale) are frequently used, but we think that morphology and not only colour and intensity (luminance) should be considered to obtained reliable algorithms in immunohistochemistry. JPEG compressed images are currently being used and they are suitable for mist applications.

We have found especially useful the automatic image evaluation of HER-2 using a combined method of immunohistochemistry analysis and CISH HER-2 test (Fig. 1).

Several experiences of TMA analyses using virtual slides have been reported. Digital reviews may solve the potential problems resulted from reviewing TMAs with a conventional microscope, such as removal of background lighting variance and dye, and potential disorientation of the reviewer [9]. Currently available TMA scoring image analysis systems are: 3DHistech Mirax TMA, Alphelys Spot Browser ${ }^{\circledR}$ 3, Aperio TMALab $^{\text {TM }}$ II, Bacus TMAscore, Dako ACIS® III
Tissue Micro-Array, Definiens TissueMap, and SlidePath OpTMA, amongst others.

TMA FISH scanning and analysis based in fluorescence microscopy is useful for high throughput cell to evaluate FISH slides [10]. Confocal laser scanning microscopy with multiple labelling TMA fluorescence can also be applied to paraffin-embedded sections [11].

Polarization filters can also be used in virtual microscopy. We have used polarization filters in an Olympus .slide system to generate virtual slides stained with Congo Red to make easier the amyloid deposits detection.

Multispectral imaging is becoming important in immunohistochemistry analysis. The optical properties of the chromogens affect the linearity and dynamic range of the assay. Thus, DAB stain exhibits scattering behaviour, while Vector Red has been shown to have excellent linearity and dynamic range. In practice, it has proven extremely difficult to unmix brown from red from blue. If we need to separate the chromogen (typically DAB) from the counterstaining (typically haematoxylin), the best approach would be multispectral imaging. This technique multiplexing capacity allows unmixing of three or more chromogens in multicolour IHC [2].

Due to the large amount of information generated, mainly in TMA analysis, some researchers have pro- 
posed an integrated solution including clustering of different assays in a platform combining clinical data, histology diagnosis, imaging, IHC and FISH data. TMA-Combiner is a solution proposed by Chih Long Liu et al (2005) at the University of Stanford [12].

In conclusion, we think that a universal pathology image based in Digital Imaging and Communications in Medicine (DICOM) standard for virtual slides will allow a rapid growth of digital (virtual) slides technology applications, including image analysis.

Consensus is needed in image analysis data and TMA data exchange. The Integrating the Healthcare Enterprise (IHE) initiative is an appropriate group to deal with this work.

High-throughput analysis is nowadays possible using digital slides (whole slide images). New scanners will become faster and efficient also in fluorescent, polarized light and multispectral imaging.

Acknowledgements: This work has been carried out with the support of COST Action IC0604.

\section{References}

[1] Cregger M, Berger AJ, Rimm DL. Immunohistochemistry and quantitative analysis of protein expression. Arch Pathol Lab Med. 2006;130:1026-30.

[2] Taylor CR, Levenson RM. Quantification of immunohistochemistry-issues concerning methods, utility and semiquantitative assessment II. Histopathology 2006;49:411-24.

[ 3] Bast RC Jr, Lilja H, Urban N, Rimm DL, Fritsche H, Gray J, et al . Translational Crossroads for Biomarkers. Clin Cancer Res. 2005;11:6103-8.
[4] Gustavson MD, Bourke-Martin B, Reilly DM, Cregger M, Williams C, Tedeschi G, et al . Development of an Unsupervised Pixel-based Clustering Algorithm for Compartmentalization of Immunohistochemical Expression Using Automated QUantitative Analysis. Appl Immunohistochem Mol Morphol. 2009 Mar 21. [Epub ahead of print].

[ 5] Masmoudi H, Hewitt S, Petrick N, Myers K, Gavrielides M. Automated Quantitative Assessment of HER-2/neu Immunohistochemical Expression in Breast Cancer. IEEE Trans Med Imaging. 2009 Jan 19. [Epub ahead of print].

[6] Rojo MG, Garcia GB, Mateos CP, Garcia JG, Vicente MC. Critical comparison of 31 commercially available digital slide systems in pathology. Int J Surg Pathol. 2006;14:285-305.

[7] Yagi Y. Critical comparison of digital pathology systems. Bioimagene Web site. 2007. Available at: http://www.bioimagene.com/pdfs/Study\%20Yagi\%20et\%20al\%20070501.pdf. Accessed February 16, 2009.

[ 8] Camp RL, Dolled-Filhart M, King BL, Rimm DL. Quantitative analysis of breast cancer tissue microarrays shows that both high and normal levels of HER2 expression are associated with poor outcome. Cancer Res. 2003;63:1445-8.

[ 9] Conway C, Dobson L, O'Grady A, Kay E, Costello S, O'Shea D. Virtual microscopy as an enabler of automated/quantitative assessment of protein expression in TMAs. Histochem Cell Biol. 2008;130:447-63.

[10] Tubbs RR, Swain E, Pettay JD, Hicks DG. An approach to the validation of novel molecular markers of breast cancer via TMA-based FISH scanning. J Mol Histol. 2007;38:141-50.

[11] Robertson D, Savage K, Reis-Filho JS, Isacke CM. Multiple immunofluorescence labelling of formalin-fixed paraffinembedded (FFPE) tissue. BMC Cell Biol. 2008;9:13.

[12] Liu CL, Montgomery KD, Natkunam Y, West RB, Nielsen TO, Cheang MC, et al . TMA-Combiner, a simple software tool to permit analysis of replicate cores on tissue microarrays. Mod Pathol. 2005;18:1641-8.

Submitted: 11 April, 2009 Accepted after reviews: 5 June, 2009 